\title{
Physical Therapy Rehabilitation Science is indexed in Korea Citation Index
}

\section{Yijung Chung}

\author{
Editor-in Chief, PTRS
}

Dear colleagues and associates of our journal, it is our greatest pleasure to announce that Physical Therapy Rehabilitation Science (PTRS) has successfully fulfilled the criteria for the Korean Citation Index (KCI) in this year of 2017. PTRS has been launched in 2012, has been publishing journals in English biannually, and has established an open access online submission system. Since then, we have received a DOI, and the references of our articles have been hyperlinked. This journal was supported by the Korean Federation of Science and Technology Societies Grant (KOFST) funded by the Korean Government. In 2014, PTRS has participated in ScienceCentral, which is a free or open-access, full-text archive of scientific society journal literature and the original text of this journal was also provided on Google Scholar, which has allowed us to raise the influence index internationally. PTRS was published quarterly in March, June, September, and December in 2016, and has added Crossmark and Fund ref functions to provide authors with information on publication.

Currently, PTRS is indexed in KCI, Google Scholar, ScienceCentral, Korean Studies Information Service
System (KISS), Research Information Sharing Service (RISS), National Digital Science Library (NDSL), KoreaScience, and DOI/Crossref.

Since this is an academic journal published in English, the research papers that have been submitted from several foreign researchers from different countries, such as the USA, Japan, Hong Kong, Saudi Arabia, etc. We are delighted by that fact that many articles of PTRS have been recently cited in Scopus and Science Citation Index journals from various countries.

In the future, we are looking forward to PTRS in becoming indexed in PubMed Central and Scopus. We will endeavor to publish high-quality articles in PTRS and will continue to strive to become a global academic journal in order to promote excellence in physical therapy and rehabilitation science internationally. Many thanks to all who have contributed to this honorable success. I would like to particularly thank the members of the editorial board who have promoted the advancement in quality of our journal and would also like to thank all of our authors and associates. 